Meta

Journal des traducteurs

Translators' Journal

\title{
Pertinence sociale de la traductologie ?
}

\section{Yves Gambier}

Volume 50, numéro 4, décembre 2005

Pour une traductologie proactive - Actes

For a Proactive Translatology — Proceedings

Por una traductología proactiva - Actas

URI : https://id.erudit.org/iderudit/019839ar

DOI : https://doi.org/10.7202/019839ar

Aller au sommaire du numéro

Éditeur(s)

Les Presses de l'Université de Montréal

ISSN

0026-0452 (imprimé)

1492-1421 (numérique)

Découvrir la revue

Citer cet article

Gambier, Y. (2005). Pertinence sociale de la traductologie ? Meta, 50(4).

https://doi.org/10.7202/019839ar

\section{Résumé de l'article}

Notre présentation s'articule autour de cinq axes: la traductologie, encore à la recherche de sa voix tout en suivant diverses voies (1), ne peut opposer théorisation et pratique (2) ; elle gagnerait sans doute aussi à peser les apports de la recherche engagée (Action Research) (3) et à s'interroger sur la pertinence sociale de ses travaux (4). L'ensemble de ces réflexions nous amène alors à proposer un réseau internationale de traductologues (5).
Ce document est protégé par la loi sur le droit d'auteur. L'utilisation des services d’Érudit (y compris la reproduction) est assujettie à sa politique d'utilisation que vous pouvez consulter en ligne.

https://apropos.erudit.org/fr/usagers/politique-dutilisation/ 


\title{
Pertinence sociale de la traductologie ?
}

\author{
YVES GAMBIER \\ Université de Turku, Turku, Finlande \\ gambier@utu.fi
}

\begin{abstract}
RÉSUMÉ
Notre présentation s'articule autour de cinq axes: la traductologie, encore à la recherche de sa voix tout en suivant diverses voies (1), ne peut opposer théorisation et pratique (2) ; elle gagnerait sans doute aussi à peser les apports de la recherche engagée (Action Research) (3) et à s'interroger sur la pertinence sociale de ses travaux (4). L'ensemble de ces réflexions nous amène alors à proposer un réseau internationale de traductologues (5).
\end{abstract}

\begin{abstract}
Five perspectives are offered in this paper: Translation Studies (TS), following different ways but still looking for its own path (1) does not oppose theory and practice (2); it would certainly benefit from Action Research (3) and from questioning its social relevance (4). All these justify the proposal to set up an international network of TS scholars (5).

\section{MOTS-CLÉS/KEYWORDS}

engagement, pertinence sociale, réseau, théorie, traductologie

Les travaux en traductologie vont bon train: ils sont nombreux, diversifiés dans leur visée et leur méthodologie ; ils trouvent assez facilement à se diffuser, au moins auprès des universitaires, grâce à des supports papier ou en ligne. Toutefois, cette circulation relative des idées, des hypothèses, des modèles, des résultats n'implique pas une reconnaissance facile, publique. La traductologie est-elle visible et lisible au-delà de ses cercles restreints ? A-t-elle des enjeux sociétaux qui donnent de la force et de la présence à certains savoirs établis, à certains domaines de recherche ?
\end{abstract}

\section{Des tournants ou des hésitations?}

Peut-on avoir un regard distancé, objectif sur les développements de la traductologie de ces deuxtrois dernières décennies ? Certainement, elle a accru sa teneur comme champ d'étude, à la fois par la variété des domaines aujourd'hui analysés, discutés (récemment introduites par exemple, sont la localisation, la traduction audiovisuelle, l'interprétation de communauté), et par son institutionnalisation (nombre de revues ont été lancées dans les années 90, comme The Translator 1995, Interpreting 1996, Across Languages and Cultures 2003 ; les départements ou instituts universitaires de traduction se sont multipliés, partout sur cette planète - de la Chine à l'Estonie, du Nigéria aux Emirats Arabes Unis ; des écoles doctorales qui se veulent internationales fonctionnent désormais en Allemagne, au Royaume-Uni, en Espagne, etc.; des associations de chercheurs ont été créées $(\S 5)$, etc.). Et pourtant si on considère les titres de conférences, d'ateliers, de séminaires, on peut être saisi d'un doute : que de répétitions, de redites, de reprises, comme si l'interdisciplinarité restait souvent une incantation, y compris entre recherche en traduction et recherche en interprétation (Schäffner 2004), comme si les langues de travail observés demeuraient toujours les mêmes, comme si l'ouverture à la traductologie dans certains pays se réduisait au mimétisme des références et réflexions bien établies.

La traductologie a connu bien des tournants depuis une quinzaine d'années (tournant culturel, idéologique, post-moderne, sémiotique, cognitif, sociologique, voilà que d'aucuns annoncent le retour de la linguistique, avec les corpus électroniques), tournants qui donnent un peu le tournis, comme si cette boulimie de virages, de tours et détours, cette vitesse pour changer d'orientations étaient plutôt une conduite en état d'ivresse, alors que dans le même temps perdure le 
souci, parfois inquiet, d'une reconnaissance par l'université. L'association européenne de traductologie (EST) a reflété cette avancée un peu tortueuse, en intitulant son $3^{\text {ème }}$ Congrès en 2001 Claims, Changes and Challenges in Translation Studies (Hansen et als. 2004) puis son $4^{\text {ème }}$ Doubts and Directions. Cependant avec les technologies de l'information et de la communication (TIC), l'intégration européenne, la globalisation et la nouvelle géographie des marchés de la traduction, les normes de qualité, le domaine et la profession bougent, acquièrent de la visibilité. Et à un moment où dominent les critères de compétitivité, de productivité, de flexibilité, de mobilité... on note la réémergence de préoccupations éthiques (Pym 2001). Alors ? Plus ça change, plus c'est pareil ? (cf. 1'intervention de Kiraly à propos de formation). Déjà Vermeer (1994: 3-6), cherchant à décrire l'état des lieux, était assez fataliste sur les notions de progrès et de traduction. Si le progrès n'est jamais rectiligne, linéaire mais davantage en zigzag, en spirale, par sauts, la traduction n'est jamais simple à définir. Vermeer et les autres adeptes du Skopos, si dominants alors, aussi dans la sélection de ces Actes de 1994, ont été renvoyés depuis à leur méditation car la suite de tournants n'échappe pas non plus à des effets de mode : ainsi ont pu un moment piloter en apparence la traductologie le modèle Vinay-Darbelney, la théorie interprétative, la théorie du Skopos, le TAP (verbalisation concourante), l'agenda à la Venuti, l'appproche par corpus, la perspective dite cognitive, etc.

Aujourd' hui, Meta en son 50 ème anniversaire (1955-2005) nous invite à regarder le futur, à anticiper l'avenir. C'est (c'était ?) une des missions en effet de l'université et des revues savantes de lier rétrospection et prospection, pour orienter les changements et non les subir, de penser nos lacunes et limitations, de prévoir, comme en météorologie et en économie, selon des indicateurs fiables et de prédire, comme en volcanologie et en sismologie, selon des modèles et des séries de données. Cet anniversaire n'est donc en rien une lecture dans le marc de café ni une bouffée de nostalgie. Il n'est pas non plus un appel à l'hyperactivisme et au zapping !

\section{Des nécessités de théoriser}

Un des premiers articles que j'ai soumis, en 1984, à Meta (Gambier 1986) a porté sur le faux dilemme qui oppose souvent théorie, résultat supposé de la tour d'ivoire, et pratique, forcément porteuse de sagesse (Chesterman \& Wagner 2002). Je n'en changerai pas un iota aujourd'hui, même si le continuum suggéré s'est depuis peut-être complexifié, avec la localisation, le doublage, l'audio-description, la rédaction technique, la téléconférence, la traduction multimédia. On sait bien que ceux qui questionnent: A quoi sert la théorie? ont presque toujours une réponse toute prête (à rien) - souvent en présupposant des dichotomies tranchées du genre contenu/forme, traduction littérale/libre, invisible/visible, approche descriptive/post-moderne, etc. Il faut dire que la traduction est encore assez fréquemment considérée comme un chemin de traverse auquel on peut aboutir à partir d'autres occupations (enseignement, écriture, journalisme, etc.) et non comme un métier avec compétences et éthique. Et puis face à la théorisation et ses multiples tournants, on peut douter de ses objectifs, de ses priorités, être aveuglé par sa diversité sinon son hétérogénéité, sa possible fragmentation (Delabastita 2003), quitte même à verser dans un scepticisme bon teint sous prétexte de la pluralité des modèles, des explications (Chesterman 1995). C'est méconnaitre le statut épistémologique des sciences humaines dont les méthodes ne peuvent transmettre la vérité d'une proposition à la suivante comme dans une chaine déductive. Leurs données d'observation, de description ne permettent ni une démonstration logico-mathématique ni un raisonnement expérimental. Selon les «cas», leurs mesures, leurs présomptions explicatives, leurs généralisations conceptuelles sont inégalement probantes et leurs intelligibilités sont inégalement fortes, en fonction de la pertinence des matériaux rassemblés et en fonction de l'argumentaire développé.

Le faux dilemme repose donc sur nombre de malentendus, touchant à la fois la pratique (réduite à une sorte de concret quotidien, à des routines et des automatismes, à des recettes) et la théorie (ne pouvant tout expliquer, elle n'expliquerait rien; diverse, elle serait amalgamée à la spéculation, abstraite nécessairement). Ce rejet éventuel de la théorie fait partie de l'idéologie du traducteur rétif, de sa «théorie» spontanée sur son travail, sa position, son rôle. L'approche sociologisante tente aujourd'hui de saisir cette résistance. 
Pourquoi la théorisation est-elle «pratique» dans ce qu'elle aide à comprendre, à expliquer, à prédire ? On peut avancer plusieurs raisons, sans souci de les hiérarchiser:

- pour systématiser les processus de traduction, à la fois en vue d'appréhender leur spécificité par rapport à d'autres productions verbales (écrites/orales) et en vue d'aider à la formalisation utile à l'automatisation. L'expertise raisonnée des traducteurs et des traductologues pourraient alors orienter les travaux en technologie de la langue - le rire jaune des uns et des autres sur la performance des outils informatisés relevant plus de la défensive que de la prospective.

- Pour saisir les décisions et choix des agents, depuis le donneur d'ouvrage jusqu'au réviseur, que ce soit au niveau global (pour une traduction cibliste, domestique, fidèle, littérale, etc.) ou au niveau local (sur les statégies à suivre pour tel ou tel segment ou problème).

- Pour comprendre les critères et procédures de qualité, d'acceptabilité des traductions c'est-à-dire pour comprendre les fonctions, les places, les représentations de la traduction et des traducteurs dans une société donnée, à un moment donné, dans l'ensemble des échanges socio-symboliques. Une telle exigence aurait des retombées par exemple sur la pré- et la post-édition en traduction automatique, sur le développement des langues contrôlées, sur la division du travail, etc.

- Pour promouvoir l'activité et le statut du traducteur.

Toutes ces raisons ont leur prolongement sur les objectifs et moyens de la formation.

Ces ambitions n'excluent pas que la recherche n'est pas forcément adaptée dans ses visées et son fonctionnement. En d'autres termes, les façons de faire de la recherche correspondent-elles aux transformations actuelles de la demande et de l'offre en traduction? (Gambier 2001-2: 19-25). De fait, la masse des travaux traductologiques aujourd'hui semble être menée par des individus isolés, est l'objet de nombreuses publications comme s'il y avait plus d'auteurs que de lecteurs, est souvent répétitive dans les sujets abordés, les données collectées, les inférences et conclusions tirées. Les paradigmes de recherche devraient donc évoluer dans le choix des thèmes à approfondir, dans les méthodes (on note déjà le besoin de créer des ressources collectives, par ex. par des corpus informatisés), dans les manières de travailler en développant des réseaux (comme celui mentionné entre Caen et Corfou, en psychologie cognitive et traduction) pour atteindre à une recherche plus collaborative et intégrant divers agents, diverses pratiques. D'où la question du rapport entre recherche et action, sinon engagement.

Une théorisation pratique, une organisation de la recherche repensée amènent à s'interroger bien évidemment sur l'identité et le statut du chercheur lui-même qui peut être et informant (traducteur) et chercheur (traductologue), c'est-à-dire juge et partie. De fait, l'exigence d'autoréflexion ne peut être sous-estimée (Gambier, à par.). Mais cette situation paradoxale n'est pas propre à la traductologie, transdiscipline réflexive dont l'épistémologie est coextensive au discours de recherche qu'elle tient (cf. Buzelin 2004; Bourdieu 1987, 2001, 2004).

\section{Pour une recherche active}

En misant sur une recherche active, il ne s'agit pas de relancer une quelconque polémique sur l'engagement (mot controversé car sans doute politisé et chargé d'histoire), même quand on célèbre le centenaire de la naissance des frères ennemis: J.P. Sartre et R. Aron, pamphlétaire de 1'Opium des intellectuels et essayiste du Spectateur engagé (1981, réédité en 2005).

On définira cette recherche engagée ou Action Research, comme un processus démocratique, participatif, liant action et réflexion, théorie et pratique, en vue d'apporter des solutions, des changements dans une organisation, un programme, une communauté (Reason \& Bradbury 2001:1). L'effort ne consiste pas à plaquer un savoir construit sur une action ni d'appliquer ce savoir à tout prix pour en faire une recherche «intéressée» mais d'informer l'un par l'autre, selon une logique où le besoin de comprendre assiste une action et où celle-ci donne forme et sens à celui-là. Dans ce processus, le chercheur dresse un plan de travail, collecte des données, analyse, en partenariat avec ceux qu'il observe, dans un dialogue permanent. Dès lors, l'objet de recherche n'est jamais isolé, détaché de ses agents (analystes et informants). Le chercheur est engagé non dans le sens d'un combat manichéen mais parce que soucieux de comprendre les retombées de sa réflexion et d'orienter sa réflexion vers certaines retombées. 
Un tel type de recherche active qui n'aboutit pas seulement à des publications a des racines déjà anciennes, par exemple en éducation et pédagogie (avec Paolo Freire), en sciences sociales (avec certains membres de l'Ecole dite de Francfort, avec Jürgen Habermas). Mais il est hors de question de retracer ici origines et développements de cette approche (parfois désignée comme épistémologie, ou comme méthode, ou comme paradigme, etc.), comme il est hors de notre propos de souligner lieux et disciplines qui y ont recours (Cooke \& Cox 2004). Qu'il suffise de dire que l'Action Research ouvre les laboratoires, les universités à d'autres partenaires, et présuppose une préoccupation partagée pour un problème, un projet, une réforme. Elle a été signalée comme perspective possible en traductologie par exemple par Kiraly (2000), Hatim (2001), Williams \& Chesterman (2002). Une thèse de doctorat a été achevée récemment (décembre 2004) par Joselia Neves sur le développement du sous-titrage pour sourds et mal entendants: son travail a impliqué participation, collaboration, responsabilisation, connaissances, changement social. ..de la part de la communauté des sourds du Portugal, d'une compagnie de télévision, de sous-titreurs professionnels, d'universitaires, dans la mesure où il n'existait pas de sous-titrage intralinguistique dans le pays, de spécialistes qualifiés, d'équipements adéquats (salles de cinéma, logiciels de soustitrage). La recherche négociée de bout en bout (2003-4) a débouché sur la résolution de ces problèmes : réalisation et moyens de diffusion de tels sous-titres, avec leurs dimensions technique et financière, formation appropriée, rédaction de directives nationales aux sous-titreurs, etc. Un tel projet qui a mobilisé jeunes chercheurs, clients (distributeurs et diffuseurs) et récepteurs a permis en moins de deux ans de faire face aux défis légaux et sociaux de l'accessibilité des moyens audiovisuels à une frange de la société - manière d'affirmer que traduction et traductologie sont des activités sociales de résolution de problèmes dont l'influence ne serait plus verticale ( $\mathrm{d}^{\text {'où le }}$ dualisme supposé entre théorie et pratique) mais horizontale, engageant des praticiens, des universitaires, des usagers.

À un moment où nombre de formateurs plaident pour un travail d'équipe, reconnaissant la division des rôles et des tâches entre producteurs, éditeurs, ingénieurs, traducteurs, réviseurs, etc., la recherche active peut certainement accélérer la construction et le développement de ces compétences de coopération, de négociation, d'auto-critique, d'écoute, de pensée partagée. Elle permet aussi de dépasser les (pseudo) oppositions, par exemple entre l'approche descriptive (critique ou pas) et les approches dites engagées (committed approaches) : post-coloniales, féministes, culturalistes (Brownlie 2003), ainsi que l'idéalisme activiste (Tymoczko 2000: 23). Quoi qu'il en soit, on doit noter la convergence des convictions que la visée du traductologue n'est plus seulement de décrire et d'expliquer mais aussi d'essayer de changer les situations (par ex. touchant le rôle et la place des traductions, les politiques de traduction, les rapports de force entre langues et cultures - Cronin 2004: 134), d'offrir des solutions à certains problèmes (par ex. touchant les relations interculturelles, le statut des traducteurs). Ce qui amène à s'interroger sur quoi agir et quand.

\section{Pertinence sociale de la traductologie}

La traduction est un phénomène social, c'est-à-dire une activité initiée et contraintes par des agents sociaux, avec des fonctions et des retombées socialement déterminées. La recherche active permet de lier projets, résultats d'analyse et besoins de traduction, face aux défis technologiques. On peut désormais situer la pertinence sociale de la traductologie à au moins trois niveaux.

a. Dans la définition des objectifs assignés à tel ou tel projet : utile pour qui ? les décideurs dans les affaires, les administrations, les services ? les juges, les officiels de l'immigration ? les autorités des industries audiovisuelles et culturelles ? Utile pour maintenant ou pour demain ? Avec quels effets à moyen et long terme ?

b. Dans le programme même de la recherche et son organisation : choix des partenaires, engagement des chercheurs. Que peut expliquer une théorisation coupée de toute pratique (traditionnelle ou nouvelle) ? Comment peut-elle prendre en considération les formes de travail émergentes et comprendre par exemple les représentations qui continuent de s'attacher à la traduction - comme par ex. qu'elle est affaire de dictionnaire, que tout natif peut traduire, qu' un texte qui a pris des jours pour être finalisé peut être traduit pour le lendemain, qu'une traduction de haute spécialité n'est pas différente d'une version faite au collège, etc. De telles représentations ne 
cessent d'occulter la traduction professionnelle, ainsi d'ailleurs que le volume de traduction par défaut, réalisé par ceux qui s'auto-traduisent vers ou de l'anglais. Non seulement il faut créer des ressources communes (ex. corpus numérisés), développer des équipes et réseaux pluridisciplinaires et pluri-institutionnels mais aussi articuler les programmes, les priorités de recherche : il y a toujours plus de thèses en traduction littéraire qu'en traduction multimédia (transfert dans les jeux vidéo, les sites web) ; il y a davantage de lieux de formation mais les enquêtes sur l'évolution des marchés des métiers langagiers font toujours cruellement défaut.

c. Dans la dissémination, la diffusion des résultats. Deux questions majeures interreliées se posent ici. D'une part, quel est le type de discours employé avec nos pairs, envers les traducteurs professionnels, vis-à-vis du public en général ? D'autre part, quelle est l'accessibilité des fruits de nos travaux ? Elle ne se réduit pas à la parution de quelques anthologies et dictionnaires encyclopédiques, utilisés par les étudiants. Elle conditionne à la fois l'interface avec d'autres disciplines (sociologie, histoire culturelle, anthropologie, psychologie cognitive, etc.) et la visibilité de nos efforts. Les réponses à ces questions n'oblitèrent pas l'exigence de réinterroger la carte de la traductologie, vu son essor récent dans différentes directions (cf. intervention de Luc Van Doorslaer) ni non plus de s'intéresser à la traduction des théories de la traduction si on ne veut pas qu'à une lingua franca corresponde l'imposition d'un mode unique de pensée.

\section{Pour un réseau international de traductologues}

Tout ce qui précède justifie la proposition qui suit. Depuis deux ans, j'ai déjà eu l'occasion de la présenter, sans qu'une stratégie de réalisation réussise jusqu'à ce jour à se mettre en place. La réforme des études universitaires dans le cadre de la Déclaration de Bologne qui visait à l'harmonisation des formations dans une quarantaine de pays européens m'amène à la relancer, parce que cette réforme a eu lieu à l'intérieur de frontières nationales, tandis que les discours institutionnels ne cessent d'insister sur la globalisation. Les traductologues et les responsables de formation continuent de penser et de décider dans le cadre des Etats nationaux, comme d'ailleurs les Associations de traducteurs - comme quoi réflexion théorique et pratique ne sont pas toujours aussi éloignées l'une de l'autre qu'on le pense ou l'affirme.

Comment est-il possible qu'un domaine connecté à l'internationalisation, à l'interculturalité, aux contacts de langues, aux TIC, reste ainsi uniquement organisé au niveau local ou régional (regroupements comme CATS/ACT - Association canadienne de traductologie, EST - European Society for Translation Studies (depuis 1992), Abrapt (au Brésil, depuis 1992), AIETI pour la péninsule ibérique (depuis 2003), ATSA pour les Etats-unis (depuis 2003), etc.). Mais dans le même temps, des associations spécialisées internationales voient le jour en localisation (LISA/Localisation Industry Standards Association), en traduction automatique (IAMT/International Association for Machine Translation, EAMT/European Association for MT), en terminologie (ELRA/European Language Resources Association) etc. On est donc témoin à la fois d'une expansion de la traductologie et d'une certaine fragmentation dans son organisation. Cette fragmentaion est en partie due à des contraintes et à des motivations d'ordre linguistique et culturelle. Elle est aussi en partie due aux différences dans le développement de la traductologie selon les zones géo-linguistiques. N'empêche, les défis ne sont plus exclusivement locaux: ils se recoupent partout, que ce soit les défis de la formation, les défis de la visibilité et de la reconnaissance (accréditation), les défis de la communication multilingue, les défis des retombées de la recherche, les défis de la technologie sur les modes et les divisions du travail, etc. Les associations locales ou transrégionales ont certainement leur raison d'être, connaissant bien ainsi les besoins et disponibilités de leurs membres, les contextes et conditions pour ajuster leurs moyens à leurs objectifs. Il ne s'agit pas d'y substituer une organisation du même genre, prétendant les coiffer toutes, selon un organigramme traditionnel qui très vite n'a que le souci de se perpétuer, selon des procédures d'auto-sélection hélas! déjà bien connues. Une organisation de ce type serait une réponse conventionnelle aux défis actuels. D'où la proposition de créer un réseau d'associations, une fédération flexible, légère, internationale, non pas imposée d'en haut mais émanant d'un consensus de ces associations locales et régionales, et utilisant les TIC qui ont accéléré le processus de globalisation. 
Quels seraient les objectifs possibles de ce réseau mondial?

- Établir une éthique et déontologie des métiers de la recherche, en particulier en traductologie, avec des actions coordonnées contre toute forme de censure et contre toute violation des droits de la personne, pour promouvoir partout la liberté de parole, la liberté d'association, la liberté académique, le libre mouvement des idées et la responsabilité des chercheurs, notamment dans leur rapport au terrain, aux partenaires et aux tiers.

- Représenter la traductologie auprès des institutions internationales et organisations non gouvernementales qui veulent maintenir et développer le multilinguisme.

- Conseiller tous ceux qui souhaiteraient mettre sur pied une association régionale de traductologie. - Soutenir chercheurs, enseignants et praticiens en difficulté (par exemple enseignants en Palestine, jeunes interprètes menacés dans la plupart des pays en guerre). Les reporters sans frontières savent se mobiliser pour défendre un des leurs, tandis que nous laissons sans voix les «language assistants» (hier dans les Balkans, en Afghanistan, en République démocratique du Congo), les «fixeurs» (aujourd'hui en Irak). Ce serait aussi l'occasion encore une fois de confirmer que réflexion et pratique ne sont pas séparées et œuvrer de concert avec d'autres associations mobilisées (comme par ex. le comité FIT pour les droits humains).

- Aider à la mobilité et à la coopération dans la formation des formateurs et des chercheurs.

- Faciliter le réseautage de centres de recherche ou d'équipes virtuelles en traductologie et favoriser la mise en place de projets de recherche multilatéraux et multilingues, intégrant notamment des universitaires isolés.

Ces deux derniers objectifs sont l'objet d'efforts dispersés, souvent brimés parce que se heurtant à des obligations nationales.

- Promouvoir l'intercompréhension culturelle, par ex. dans les multinationales, lors des fusions d'entreprise, dans les relations internationales.

- Améliorer les normes et critères de recherche dans les programmes postgradués.

- Encourager les rencontres interdisciplinaires, aidant graduellement à la reconnaissance de la traductologie.

- Faciliter la dissémination des publications en traductologie, par exemple en développant une bibliographie réellement internationale, en faisant reconnaitre la traductologie dans les bases de données (bibliographiques, de citations).

Ces objectifs sont des suggestions car le réseau projeté ne saurait être parachuté. Il reste, ensemble, à dégager une stratégie collective qui lancerait le projet. Cela prendra peut-être du temps à cause de l'attentisme des uns ou de la prudence des autres. Il n'en demeure pas moins que les défis à relever sont là, que les processus de la globalisation ne retourneront pas en arrière.

Pour conclure, on répétera que la recherche qui n'est jamais sans valeur a des implications sociales. Le chercheur ne peut nier qu'il a sans cesse à articuler production de savoir (recherche), transmission (enseignement) et pouvoir (comme par ex. membre de jury, dans les revues, etc.). Aujourd'hui, il est de plus en plus pris dans le monde qu'il cherche à comprendre: il lui reste avec d'autres à dépasser ces rêts dans un réseau qui lui permettrait de préserver et de renforcer son autonomie.

\section{RÉFÉRENCES}

BASNETt, S. (1998) : «The Translational Turn in Cultural Studies », in S. BASNETT \& A. LeFEvere, Constructing cultures. Essays on Literary Translation, Clevedon, Multilingual Matters, p. 123-140.

Bourdieu, P. (1987) : Choses dites, Paris, Ed. de Minuit.

Bourdieu, P. (2001) : Science de la science et réflexivité, Paris, Raisons d'agir Editions.

BOURDIEU, P. (2004) : Esquisse pour une auto-analyse, Paris, Raisons d'agir Editions.

BRownlie, S. (2003) : "Distinguish Some Approaches to Translation Research: The Issue of Interpretative

Constraints", The Translator 9-1, p. 39-64.

BuZElin, H. (2004) : « La traductologie, l'ethnographie et la production des connaissances », Meta 49-4, p. 729-

746. 
Chesterman, A. (1995) : “The Successful Translator. The Evolution of Homo Transferens", Perpectives 3-2, p. 253-270.

Chesterman, A. and E. WAgner (2002) : Can Theory Help Translators? A Dialogue Between the Ivory Tower and the Wordface, Manchester, St Jerome.

Cooke, B. and J. Wolfram Cox (eds) (2004) : Fundamentals of Action Research (4 vol.), Londres, Sage.

Cronin, M. (2003) : Translation and Globalization, Londres and New York, Routledge.

Delabastita, D. (2003) : "Translation Studies for the 21 st Century. Trends and Perpectives", Génesis 3, p. 7-24.

GAMBIER, Y. (1986): «Théorie et pratique: une fause alternative. Pour un concept dynamique de la traduction »,

Meta 31-2, p. 161-172.

GAMBIER, Y. (2001-2002) : « La traductologle a-t-elle encore un avenir sans traducteurs ? », Equivalence 28-2, p. 19-29.

GAMBIER, Y. (2003) : « Les passeurs langagiers: réflexions sur les défis de la formation », in G. MARESCHAL, L. BRunetTe, Z. Guevel \& E. VAlentine (réd.) : La formation à la traduction professionnelle, Ottawa, Presses universitaires d'Ottawa.

GAMBIER, Y. (à par.) : «Y a-t-il une place aujourd'hui pour une socio-traductologie ?» Exposé lors de la conférence de Graz sur Translating and Interpreting as a social practice (5-7.5.2005).

Hansen, G, K. Malmkjaer and D. Gile (eds) (2004) : Claims, Changes and Challenges in Translation Studies, Amsterdam and Philadelphia, J. Benjamins.

Hatim, B. (2001) : Teaching and Researching Translation, Harlow, Pearson Education Limited.

KIRALY, D. (2000) : A Social Constructivist Approach to Translator Education - Empowerment from Theory to Practice, Manchester, St Jerome.

PYM, A. (2001) : "Introduction: The Return to Ethics in Translation Studies", The Translator 7-2, p.129-138.

REASON, P. and H. BRADBURY (eds) (2001) : Handbook of Action Research - Participative Inquiry and Practice, Londres, Sage.

SCHÄFFNER, C. (ed.) (2004) : Translation Research and Interpreting Research. Traditions, Gaps and Synergies, Clevedon, Multilingual Matters.

TYMoczKo, M. (2000) : "Translation and Political Engagement: Activism, Social Change and the Role of Translation in Geopolitical Shifts", The Translator 6-1, p. 23-47.

VermeER, H. (1994) : "Translation Today: Old and New Problems", in M. SNell-Hornby, F. PöchHaCKer \& K. KAINDL (eds) : Translation Studies: An Interdiscipline (Selected papers from the Congress held in Vienne, 912.9.1992), Amsterdam and Philadelphia, J. Benjamins, p. 3-16.

Williams, J. and A. Chesterman (2002) : The Map. A Beginner's Guide to Doing Research, Manchester, St Jerome.

WILSS, W. (2004) : “Translation Studies. The State of the Art”, Meta 49-4, p. 777-785. 